\title{
Implementación en FPGA de un sistema de medida de impedancia armónica de circuitos resonantes en serie basado en el algoritmo lock-in
}

\author{
O. Jiménez1, L.A. Barragánı, I. Urriza1, O. Lucía1, D. Navarro1, J.I. Artigas2 \\ ${ }_{1}$ Grupo de Electrónica de Potencia y Microelectrónica (GEPM \\ Instituto de Investigación en Ingeniería de Aragón (I3A) \\ Universidad de Zaragoza, Mariano Esquillor s/n, 50018, Zaragoza, Spain. \\ Tel.+34-976762707,Fax+34-976762043,e-mail: ojimenez@unizar.es \\ ${ }_{2}$ Tecnologías para la mejora de la calidad de vida de personas con discapacidad (TECNODISCAP) \\ Instituto de Investigación en Ingeniería de Aragón (I3A) \\ Universidad de Zaragoza, Mariano Esquillor s/n, 50018, Zaragoza, Spain.
}

\begin{abstract}
En esta ponencia se presenta un sistema digital implementado en FPGA (field programmable gate array) cuya función es la medida en tiempo real de la impedancia del primer armónico de una carga de una cocina de calentamiento por inducción. La etapa inversora de la cocina implementa la topología semipuente resonante serie de media frecuencia ( $25 \mathrm{kHz}-75 \mathrm{kHz}$ ) cuyo circuito de carga consiste en un condensador en serie con el sistema inductor-recipiente (inductor planar acoplado con un recipiente). En una primera aproximación, la impedancia del circuito puede modelarse como una red equivalente R-L-C, sin embargo, la impedancia equivalente depende de múltiples factores tales como el material del recipiente, alineamiento entre el recipiente y el inductor, temperatura o frecuencia de trabajo. Por dicha razón, una caracterización en tiempo real de la carga es deseable para realizar un correcto control de la etapa inversora. El sistema de identificación calcula la impedancia del primer armónico de la carga aplicando el algoritmo lock-in de fase dual a la corriente que atraviesa el inductor y a la tensión de salida del inversor. Dicho algoritmo ha sido descrito en VHDL (very high speed integrated circuit hadware description language) e implementado en una FPGA de Xilinx. La precisión del algoritmo ha sido verificada mediante simulación a través de una herramienta de simulación mixta (analógica-digital). Finalmente, el sistema ha sido verificado experimentalmente.
\end{abstract}

